\title{
Microbiology profile in women with pelvic inflammatory disease in relation to IUD use
}

\author{
I. VIBERGA ${ }^{1,3}$, V. ODLIND ${ }^{3}$, G. LAZDANE ${ }^{1}$, J. KROICA ${ }^{2}$, L. BERGLUND $^{4}$, \& \\ S. OLOFSSON ${ }^{4}$ \\ ${ }^{1}$ Department of Obstetrics and Gynaecology, Uppsala University, Uppsala, Sweden, ${ }^{2}$ Department of Microbiology, Riga \\ Stradins University, Riga, Latvia, ${ }^{3}$ Department of Women's and Children's Health, International Maternal and Child \\ Health, and ${ }^{4}$ Uppsala Clinical Research Centre, UCR, Uppsala University, Uppsala, Sweden
}

\begin{abstract}
Objective. To study the microbial characteristics of patients with pelvic inflammatory disease (PID) and the possible impact of an intrauterine device (IUD) on the microbial environment in women presenting with PID.

Methods. Case-control study, investigating 51 women with acute PID and 50 healthy women. Endocervical specimens for microbiological investigation were obtained at gynaecological examination.

Results. IUD users with PID had significantly more Fusobacteria spp. and Peptostreptococcus spp. than non-IUD users with PID. The finding of combinations of several anaerobic or aerobic microbes was associated with a significantly increased risk of PID and with complicated PID. In IUD users, the combinations of several anaerobic/aerobic microbes were associated with an increased risk of PID, irrespective of duration of IUD use. Long-term IUD use appeared to be associated with an increased risk of a PID being complicated.

Conclusion. The finding of several anaerobic or aerobic microbes appears to be associated with PID in users of IUD.
\end{abstract}

Keywords: Pelvic inflammatory disease, intrauterine device, microbiology, anaerobes

\section{Introduction}

Pelvic inflammatory disease (PID) is the most common gynaecological infection. PID is associated with a high morbidity resulting from an inflammation and damage of the reproductive tract that can lead to sequelae such as tubal factor infertility, ectopic pregnancy and chronic pelvic pain [1,2]. Many studies have been carried out during the last four decades to determine the microbiological aetiology of PID. Mainly, they demonstrate a direct association between PID and sexually transmitted infections (STI) such as Chlamydia trachomatis and Neisseria gonorrhoeae [1-4]. It is well established that the highest PID and STI prevalence is found in sexually active women below 25 and is directly related to the sexual behaviour of both partners $[1,2,4-6]$. However, the bacterial pathogenesis of PID is unclear in cases where STI microbes are not detected, and where behavioural correlates of PID are not apparent. Many authors mention the role of facultative and anaerobic pathogens [4,5,7-10], as well as Mycoplasma hominis and Ureaplasma urealyticum [11], as aetiological factors in PID.

There is limited knowledge about the risk of PID attributable to the use of an intrauterine device (IUD). The use of IUDs and risk of PID has been a topic for discussions between clinicians and researchers ever since IUDs were introduced. In particular, the possible influence of an IUD on the microbiological findings in the female genital tract and the impact of an IUD on the ascension of pathogens and on the severity of PID have been extensively discussed [4,12-14]. Following the concern associated with the 'Dalkon shield' type of IUD, WHO conducted a large study, and one important conclusion was that the risk of PID related to IUD use has been exaggerated [15].

According to a recent survey (1997) $20.3 \%$ of women aged 15 to 45 in Latvia use IUDs, making IUDs the most common contraceptive method used. Since the 1990s only copper releasing IUDs have 
been available in Latvia and no study on the relationship between IUD use and PID has been performed. This study was designed as a case-control study to test the following hypotheses: IUD users have a higher risk of PID than non-users, IUD users have more severe clinical characteristics of PID than non-users, IUD users have a more complicated clinical course of PID than non-users, and IUD users have different microbiological findings compared with non-users. Some descriptive results from the study have already been presented [16]. The purpose of this report is to present the microbiological findings.

\section{Material and methods}

\section{Study population and definitions}

The cases studied were women admitted to hospital with a diagnosis of acute PID. Women, who were healthy and attended outpatient clinics for routine gynaecological check-ups without any complaints, served as controls. A detailed description of the protocol, clinical criteria for PID cases, and inclusion and exclusion criteria for the study group have been presented elsewhere [16].

In brief, at the time of recruitment women had to be aged 25-45, and, if the case or control woman was an IUD user, the IUD usage had to exceed one month in order to avoid directly post-insertion related complications [13,17]. A complicated PID case was defined upon the clinical findings at admission of a palpable adnexal tumor (bilateral or unilateral) and elevated body temperature and at least one of three of the following laboratory signs: leukocyte count $\geqslant 10000 / \mathrm{mm}^{3}$, erythrocyte sedimentation rate $\geqslant 15 \mathrm{~mm} / \mathrm{h}$ or $\mathrm{C}$-reactive protein $\geqslant 20 \mathrm{mg} / \mathrm{l}$. A case was also retrospectively defined as complicated if treatment required surgery (unilateral or bilateral salpingectomy or adnexectomy).

Between December 1998 and January 2001, all 51 women admitted to Riga 1st Hospital, Dzelzcelnieku Hospital and Lags-Centrs Hospital (Riga, Latvia), who were diagnosed with acute PID according to established criteria, were asked to participate in the study by a duty gynaecologist. During the same period, among all healthy consecutive outpatient clinic visitors, 50 women, who met the inclusion and exclusion criteria, were asked to participate as controls by the principal investigator during her work hours.

The purpose of the study was presented to every eligible woman and she was asked to sign an informed consent form. Some results from the study have already been presented [16]. In summary, women with PID (cases) differed significantly from healthy controls in that they were slightly older, had spent less time in education, were more likely to be unemployed and were more often smokers. The cases also reported longer time since last pregnancy and longer use of current contraceptive method than healthy controls. However, there were no significant differences between cases and controls with regard to typical risk factors for PID such as age at first intercourse, number of sex partners, duration of current sexual relationship, number of previous pregnancies and previous episodes of PID. IUD use was common in both cases (55\%) and controls (40\%) and this difference was not statistically significant.

\section{Study methods}

All participants were asked to complete a structured questionnaire about life habits, sexual behaviour, and contraceptive use, and reproductive, gynaecological and medical histories were taken during the outpatient visit (controls) or during the hospital stay (cases). All cases were treated in-clinic.

\section{Microbiological investigation}

On admission, all women, both cases and controls, underwent a gynaecological examination, and endocervical specimens for microbiological investigation were obtained. Specimens were numbercoded according to the case/control questionnaire, collected and transported by the principal investigator. For transportation of bacteriological material, transport-media ('Amies') with charcoal was used. The transportation time did not exceed 12 hours. For identification of DNA of $N$. gonorrhoeae and C. trachomatis, material was transported in the medium 'Digene'. Material from endocervix was Gram-stained, cultured and DNA of $N$. gonorrhoeae and C. trachomatis was identified. Microbiological analysis was performed at the Department of Microbiology of Riga Stradins University and the Laboratory of Virology of a State agency, the 'Public Health Agency'. Methods used for identification of microorganisms are summarized in Table I.

\section{Statistical methods}

All data were analyzed and compared between groups using the SAS statistical package. Frequency tables were analyzed using Fisher's Exact test. Odds ratios were calculated and tested with Wald's test where appropriate. In all analyses, a $p$-value $<0.05$ was considered significant. The calculation of sample size with the statistical power $80 \%$ was based on the percentage of IUD use in Latvia's female population, and the assumption, based on clinical observation in Latvia, that approximately half of PID case women above 25 years of age use an IUD. 
Table I. Identification methods for microorganisms.

\begin{tabular}{|c|c|}
\hline Anaerobic bacteria spp.: & $\begin{array}{l}\text { bacteriological method, bacteria were incubated in Schaedler agar with } \\
5 \% \text { sheep blood in 'Genbag anaer.' with further identification } \\
\text { of biochemical properties and antigenic structure }\end{array}$ \\
\hline Mycoplasma hominis, Ureaplasma urealyticum: & bacteriological method, Mycoplasma Lyo medium, Bio-Merieux \\
\hline Trichomonas vaginalis: & bacteriological method, Trichosel broth, BBL \\
\hline Streptococcus spp.: & $\begin{array}{l}\text { bacteriological method, Columbia blood agar, Bio-Merieux and Slidex strepto } \\
\text { for identification of groups }\end{array}$ \\
\hline Staphylococcus spp.: & $\begin{array}{l}\text { bacteriological method, Columbia blood agar, Chapman's agar, Bio-Merieux } \\
\text { and Slidex staphylo for identification of } S \text {. aureus }\end{array}$ \\
\hline Candida spp.: & $\begin{array}{l}\text { bacteriological method, Sabouraud chloramphenicol agar and API } 32 \mathrm{C} \text { system } \\
\text { for yeast identification }\end{array}$ \\
\hline Actinomyces spp.: & bacteriological method, Columbia blood agar, Bio-Merieux \\
\hline Gardnerella vaginalis: & smear, Gram-stained \\
\hline Neisseria gonorrhoeae, Chlamydia trachomatis: & DNA Digene C, G test (Hybrid Capture II System), general primers GP5,6 \\
\hline
\end{tabular}

\section{Ethics}

The Ethical Committees of the Ministry of Welfare of Latvia and Uppsala University approved the study protocol.

\section{Results}

\section{The endocervical microbial profile}

The microbiological results are presented for the case group in comparison with the control group and for women with and without IUDs among cases as well as among controls (Table II). The microbiological results are shown for uncomplicated cases compared with complicated cases of PID and results are presented for those groups with regard to IUD use or non-IUD use (Table III).

STI microbes. Only one case of $N$. gonorrhoeae was found in a woman with PID and three cases of C. trachomatis were detected, all of which were found in the healthy controls.

Aerobic bacteria. There were no significant differences between healthy and ill women, between uncomplicated and complicated PID cases, or between IUD users and non-users in the PID group with regard to all detected aerobic microbes. Ureaplasma urealyticum was significantly more often detected in healthy IUD users than in healthy non-users $(p=0.034)$.

Anaerobic bacteria. Regarding anaerobic bacteria, there was no significant difference between PID women and healthy controls, or between uncomplicated and complicated cases of PID. However, IUD users among women with PID had significantly more Fusobacteria spp. $(p=0.006)$ and Peptostreptococcus spp. $(p=0.021)$ than did non-users with PID.

Facultative anaerobic bacteria. Lactobacillus spp. was significantly less frequently found in the PID group than in the control group $(p=0.013)$, but there was no significant difference between complicated and uncomplicated cases of PID or between IUD users and non-users.

\section{Synergism between microbes}

In order to test the hypothesis of whether there is a synergistic effect between several microbes in the pathogenesis of PID, the five most frequently detected microbes were included in the analyses. Among aerobic microbes Staphylococcus spp. and Streptococcus spp. and among anaerobes Bacterioides spp., Fusobacteria spp. and Peptostreptococcus spp. were included in the analysis.

Risk of PID and risk of a PID being complicated according to the endocervical microbial profile. The risk for a woman of having a PID and the risk for a woman with PID to be a complicated case according to the polymicrobial environment in the endocervix, was tested using a univariate analysis of data (Table IV). The finding of at least two anaerobic microbes was associated with a PID diagnosis $(\mathrm{OR}=2.8$; CI 1.04-7.6). Similarly, the finding of at least three aerobic or anaerobic microbes was associated with an increased risk of PID (OR $=2.4$; CI 1.04-5.9). In women with PID, the finding of at least two anaerobic microbes was associated with an increased risk of the PID being complicated $(\mathrm{OR}=4.0 ;$ CI 1.07-14.9). Stratifying for age, the association between the finding of at least two anaerobic microbes or at least three aerobic or anaerobic microbes and the risk of PID or the risk of a PID being complicated appeared stronger in women over 35. This finding was, however, not statistically significant.

Risk for an IUD user of having a PID or complicated PID according to the endocervical microbial profile. In order to assess the role of IUDs, and, in particular, 


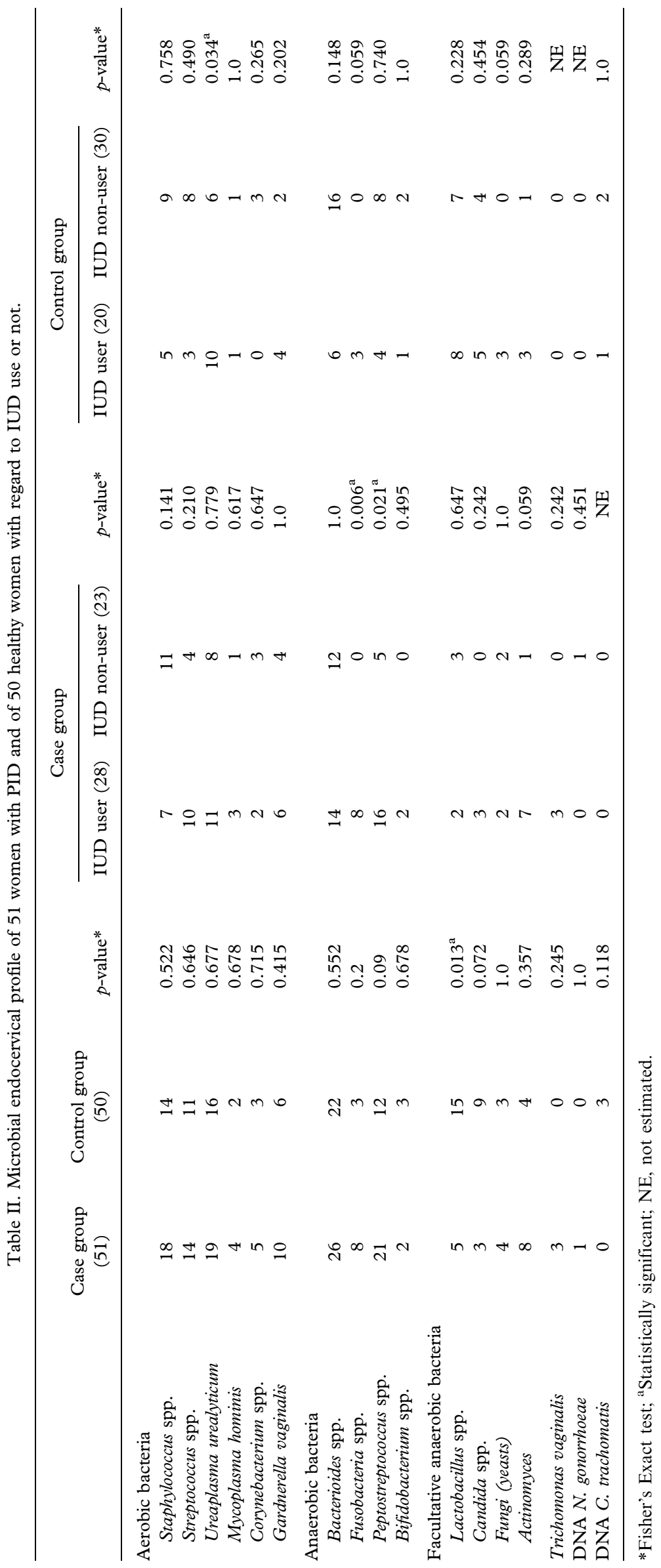




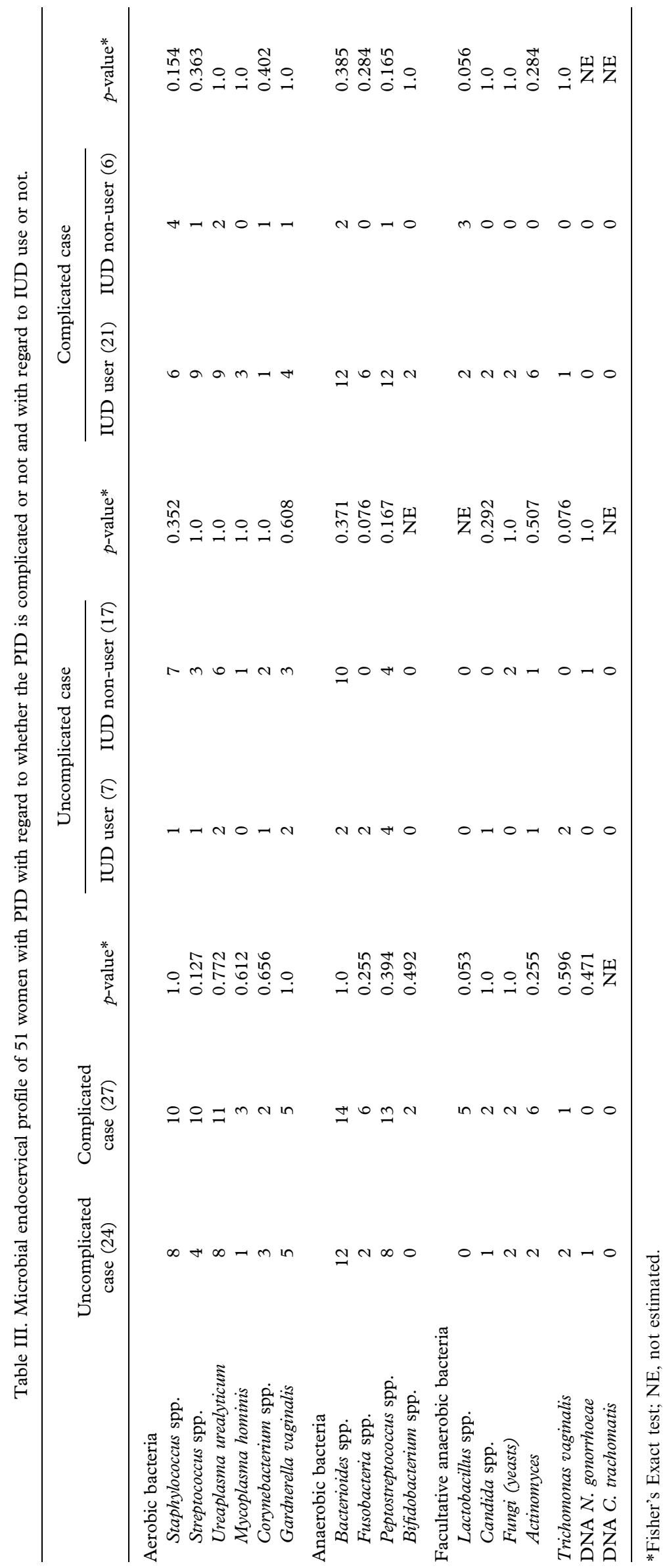


the duration of use, on the risk of PID, IUD users were analysed separately from non-users with regard to microbial findings (Table V). The finding of at least two anaerobic microbes $(\mathrm{OR}=9.0$, CI 1.8-46.3) or at least three aerobic or anaerobic microbes $(\mathrm{OR}=4.0$, CI 1.1-15.0) in an IUD user was statistically significantly associated with PID. Among IUD users with PID, no statistically significant association was found between any combination of aerobic or anaerobic microbes and the risk of having a complicated PID. Stratifying data by duration of usage of IUD ( $<5$ years and $>5$ years) did not reveal an overall higher risk in women with long ( $>5$ years) usage of IUD. However, long duration of IUD use together with the finding of at least two anaerobic microbes appeared to be associated with an increased, although not statistically significant, risk of complicated PID $(\mathrm{OR}=3.0$, CI 0.2-41.0).

Risk for an IUD non-user to have a PID or complicated PID according to the endocervical microbial profile. When IUD non-users were analysed separately with the same approach there was no statistically significant OR for PID with any combination of aerobic or anaerobic microbes in the endocervix (Table VI).

Table IV. The risk of having PID in 51 cases and 50 controls and the risk of having a complicated PID in 24 uncomplicated cases and 27 complicated cases, based on endocervical microbial profile and stratified by age groups.

\begin{tabular}{|c|c|c|c|c|c|c|}
\hline & \multicolumn{3}{|c|}{ Study group (101) } & \multicolumn{3}{|c|}{ PID group (51) } \\
\hline & OR & CI $95 \%$ & $p$-value* & OR & CI $95 \%$ & $p$-value* \\
\hline At least two anaerobic microbes & $2.8^{\mathrm{a}}$ & $1.04-7.6$ & 0.042 & $4.0^{\mathrm{a}}$ & $\mathrm{z} 1.07-14.9$ & 0.039 \\
\hline $25-34$ years & 1.6 & $0.4-7.3$ & 0.522 & 0.7 & $0.1-8.5$ & 0.796 \\
\hline$\geqslant 35$ years & 3.0 & $0.7-13.0$ & 0.143 & 8.5 & $0.9-83.1$ & 0.064 \\
\hline At least three aerobic or anaerobic microbes & $2.4^{\mathrm{a}}$ & $1.04-5.9$ & 0.041 & 1.9 & $0.6-5.8$ & 0.285 \\
\hline $25-34$ years & 1.9 & $0.6-5.8$ & 0.283 & 0.5 & $0.1-3.5$ & 0.496 \\
\hline$\geqslant 35$ years & 4.9 & $0.9-25.8$ & 0.062 & 8.6 & $0.9-83.1$ & 0.064 \\
\hline
\end{tabular}

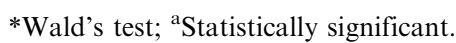

Table V. The risk of having PID among 48 women with IUD (A; 28 PID women and 20 healthy women) and the risk of having a complicated PID among 28 PID women with IUD (B; 7 uncomplicated cases and 21 complicated cases), based on endocervical microbial profile and stratified by IUD usage length.

\begin{tabular}{|c|c|c|c|c|c|c|}
\hline & \multicolumn{3}{|c|}{ A (48) } & \multicolumn{3}{|c|}{ B (28) } \\
\hline & OR & CI $95 \%$ & $p$-value* & OR & CI $95 \%$ & $p$-value* \\
\hline At least two anaerobic microbes & $9.0^{\mathrm{a}}$ & $1.8-46.3$ & 0.009 & 1.5 & $0.3-8.2$ & 0.663 \\
\hline$<5$ years & 9.3 & $0.9-101.9$ & 0.067 & 0.5 & $0.04-6.7$ & 0.6 \\
\hline$\geqslant 5$ years & 5.0 & $0.5-54.0$ & 0.185 & 3.0 & $0.2-41.0$ & 0.410 \\
\hline At least three aerobic or anaerobic microbes & $4.0^{\mathrm{a}}$ & $1.1-15.0$ & 0.04 & 3.3 & $0.5-21.3$ & 0.203 \\
\hline$<5$ years & 4.0 & $0.7-23.5$ & 0.125 & 6.0 & $0.4-101.6$ & 0.215 \\
\hline$\geqslant 5$ years & 4.0 & $0.4-43.1$ & 0.253 & 2.3 & $0.2-31.0$ & 0.534 \\
\hline
\end{tabular}

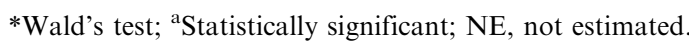

Table VI. The risk of having PID among 53 women without IUD (A; 23 PID women and 30 healthy women) and the risk of having a complicated PID among 23 PID women without IUD (B; 17 uncomplicated cases and 6 complicated cases), based on endocervical microbial profile.

\begin{tabular}{|c|c|c|c|c|c|c|}
\hline & \multicolumn{3}{|c|}{ A (53) } & \multicolumn{3}{|c|}{$\mathrm{B}(23)$} \\
\hline & OR & CI $95 \%$ & $p$-value* & OR & CI $95 \%$ & $p$-value* \\
\hline At least two anaerobic microbes & 0.5 & $0.1-2.7$ & 0.403 & 3.2 & $0.2-61.0$ & 0.439 \\
\hline At least three aerobic and anaerobic microbes & 1.4 & $0.4-4.9$ & 0.562 & 0.4 & $0.03-4.0$ & 0.406 \\
\hline
\end{tabular}

*Wald's test. 


\section{Discussion}

The decision to collect data on women with the age restriction $\geqslant 25$ was made to avoid the traditional risk factors for PID and, thus, to investigate if and to what extent IUD use per se could account for microbiological differences and the aetiology of PID. Surprisingly few cases with $N$. gonorrhoeae and $C$. trachomatis were detected. It is unlikely that this lack of common STI pathogens is due to methodological imprecision, since the method used for the analysis is highly sensitive and specific for the detection of those sexually transmitted microbes [18]. This finding might rather be explained by the absence of classical risk factors for STI in our study group and there were no difference between women with PID and healthy controls with regard to common risk factors for STI [16].

Lactobacillus spp. is the dominant microbe in the vaginal-endocervical ecosystem of healthy women and is believed to act as a defence factor inhibiting aerobic and anaerobic microbe growth and colonisation [19]. Lack of this dominance could promote overgrowth of endogenous aerobic and anaerobic bacteria and, subsequently, promote the development of PID, explaining the difference found between healthy and ill women.

Some reports have shown an association between bacterial vaginosis (BV) and IUD use [20,21], but in the present study the occurrence of BV was not investigated. The more frequent finding in IUD users of Fusobacteria spp. and Peptostreptococcus spp. is difficult to explain. Those Gram-positive anaerobic cocci are a major part of the normal human flora of the large bowel in general and of the female genitourinary system in particular. Counts are known to vary with physiological processes such as the menstrual cycle and pregnancy, which makes assessment of the normal flora complicated. The sampling in the study was not stratified for time in the menstrual cycle and only a minority of non-IUD users were on steroidal contraceptives.

There is considerable debate whether anaerobes can act as primary pathogens or only as secondary invaders when the urogenital tract is already damaged, or whether they are important components of a synergistic group of pathogens. The pathogenicity of anaerobes has been little studied. A series of experiments and a few studies have shown that most species of anaerobes express their pathogenicity via a synergistic interaction with facultative organisms or other anaerobes $[9,10]$. Anaerobic bacteria elaborate a variety of potential virulence factors, but little is known about circumstances when and why and how anaerobes cause disease $[9,10]$. There are no clear data about the prevalence and activity of anaerobes in the vaginal ecosystem in IUD users. Moreover, in studies on the microbiological profile of healthy women prior to IUD insertion, during follow-up and microbiological investigation of removed IUDs of healthy women and of women with PID, there is a diversity of detected microbes and researchers have come to different conclusions [22-25]. From the present study, it appears as if IUD use could promote the growth of anaerobes and, thus, play a role in the expression of their virulence factors in the development of PID.

From the present study it appears that synergism between combinations of microbes plays an important role in the development of PID. The study also supports the hypothesis that IUD use together with the occurrence of combinations of anaerobic/aerobic microbes may facilitate the development of PID. The impact of IUD use on the development of PID is supported by the finding that, among IUD non-users, no microbial combination affected the risk of PID. With regard to the duration of IUD use, the study could not confirm that long duration of use was a particular risk factor for PID in general, whereas long duration of use was associated with a high risk of having a complicated PID, suggesting that the duration of IUD use may still be important. There appears to be a tendency that older age $(\geqslant 35)$ increases the risk of PID, but in the present study, there were too few women over 35 to reach statistical significance.

Actinomyces are part of the normal vaginal flora, but complicated PID, associated with Actinomyces, has been reported in the literature, especially among IUD users [26]. Understanding of the pathogenesis of pelvic actinomycosis and why it correlates with IUD use is poor and controversial. In the present study, there were too few cases of Actinomyces to show a clear impact on the development of PID, although Actinomyces were found slightly more often in IUD users with PID.

A possible limitation in the study could be the selection of the control women, who were selected on the assumption that they were likely to be similar to the women with PID in most respects. Healthy women suitable to serve as controls are difficult to identify and there is no population base in Latvia where ideal controls could be found. The control women represented healthy women in general who followed the common national recommendation to have a routine gynaecological check-up and, although they differed slightly in age, both PID women and healthy controls were similar with regard to previous gynaecological morbidity and attendance to a gynaecologist during the last year as well as sexual behaviour and contraceptive use [16].

In conclusion, whereas there was little difference between groups with regard to single microbes, the finding of combinations of several anaerobic or aerobic/anaerobic microbes appears to be associated 
with PID, particularly in women over 35 . Long-term IUD use may possibly be associated with an increased risk of developing a complicated PID. The study supports the hypothesis that the pathogenesis of non-sexually transmitted PID is associated with a synergistic effect between several pathogens, possibly facilitated by the presence of an IUD.

\section{Acknowledgments}

This study was initially made possible thanks to the FIGO Fellowship (Schering Foundation) 1998/ 1999, and the Familjeplaneringsfonden I Uppsala 2000/2001 and a Swedish Institute Wisby Program Fellowship 2002 for Ilze Viberga financially supported further research. We thank Emeritus Professor Dan Danielsson, MD, PhD for assistance and advice during the preparation of this manuscript for publication.

\section{References}

1. Westrom L. Incidence, prevalence, and trends of acute pelvic inflammatory disease and its consequences in industrialized countries. Am J Obstet Gynecol 1980;138:880-892.

2. Simms I, Stephenson JM. Pelvic inflammatory disease epidemiology: what do we know and what do we need to know? Sex Transm Inf 2000;6:80-87.

3. Paavonen J, Lehtinen M. Chlamydial pelvic inflammatory disease. Hum Reprod Update 1996;2:519-529.

4. Jossens MO, Schachter J, Sweet RL. Risk factors associated with pelvic inflammatory disease of differing microbial etiologies. Obstet Gynecol 1994;83:989-997.

5. Jossens MOR, Eskenazi B, Schachter J, Sweet RL. Risk factors for pelvic inflammatory disease. Sex Transm Dis 1996;23: 239-247.

6. Lee NC, Rubin GL, Grimes DA. Measures of sexual behavior and the risk of pelvic inflammatory disease. Obstet Gynecol 1991;77:425-430.

7. Baveja G, Saini S, Sangwan K, Arora DR. A study of bacterial pathogens in acute pelvic inflammatory disease. J Commun Dis 2001;33:121-125.

8. Walker CK, Workowski KA, Washington AE, Soper D, Sweet RL. Anaerobes in pelvic inflammatory disease: implications for the Centers for Disease Control and Prevention's guidelines for treatment of sexually transmitted diseases. Clin Infect Dis 1999;28:S29-S36.

9. Murdoch DA. Gram-positive anaerobic cocci. Clin Microbiol Rev 1998;11:81-120.
10. Brook I. Microbiology and management of polymicrobial female genital tract infections in adolescents. J Pediatr Adolesc Gynecol 2002;15:217-226.

11. Arya OP, Tong CYW, Hart CA, Pratt BC, Hughes S, Roberts P, Kirby P, Howel J, McCormic A, Goddard AD. Is Mycoplasma hominis a vaginal pathogen? Sex Transm Inf 2001;77:58-62.

12. Darney PD. Time to pardon the IUD? N Engl J Med 2001;345:608-610.

13. Gareen IF, Greenland S, Morgenstern H. Intrauterine devices and pelvic inflammatory disease: meta-analyses of published studies, 1974-1990. Epidemiology 2000;11:589-597.

14. Grimes DA. Intrauterine device and upper-genital-tract infection. Lancet 2000;356:1013-1019.

15. Farley TM, Rosenberg MJ, Rowe PJ, Chen JH, Meirik O. Intrauterine devices and pelvic inflammatory disease: an international perspective. Lancet 1992;339:785-788.

16. Viberga I, Odlind V, Lazdane G. Characteristics of women presenting with PID and healthy women in Riga, Latvia. Eur J Contracept Reprod Health Care 2004 (in press).

17. Vessey MP, Yeates D, Flavel R, McPeterson K. Pelvic inflammatory disease and the intrauterine device: findings in a large cohort study. Br Med J (Clin Res Ed) 1981;282: 855-857.

18. Girdner JL, Cullen AP, Salama TG, He L, Lorincz A, Quinn TC. Evaluation of the Digene Hybrid Capture II CT-ID test for detection of Chlamydia trachomatis in endocervical specimens. J Clin Microbiol 1999;37:1579-1581.

19. Aroutcheva A, Gariti D, Simon M, Shott S, Faro J, Simoes JA, Gurguis A, Faro S. Defence factors of vaginal lactobacilli. Am J Obstet Gynecol 2001;185:375-379.

20. Ferraz do Lago R, Simoes JA, Bahamondes L, Camargo RP, Perroti M, Monteiro I. Follow-up of users of intrauterine device with and without bacterial vaginosis and other cervicovaginal infections. Contraception 2003;68:105-109.

21. Calzolari E, Masciangelo R, Milite V, Verteramo R. Bacterial vaginosis and contraceptive methods. Int $\mathrm{J}$ Gynaecol Obstet 2000;70:341-346.

22. Haukkamaa M, Stranden P, Jousimies-Somer H, Siitonen A. Bacterial flora of the cervix in women using an intrauterine device. Contraception 1987;36:527-534.

23. Elhag KM, Bahar AM, Mubarak AA. The effect of a copper intra-uterine contraceptive device on the microbial ecology of the female genital tract. J Med Microbiol 1988;25:245-251.

24. Tsanadis G, Kalantaridou SN, Kaponis A, Paraskevaidis E, Zikopoulos K, Gesouli E, Dalkalitsis N, Korkontzelos I, Mouzakioti E, Lolis DE. Bacteriological cultures of removed intrauterine devices and pelvic inflammatory disease. Contraception 2002;65:339-342.

25. Lewis R. A review of bacteriological culture of removed intrauterine contraceptive devices. Br J Fam Plann 1998;24:95-97.

26. Lippes J. Pelvic actinomycosis: a review and preliminary look at prevalence. Am J Obstet Gynecol 1999;180:265-269. 


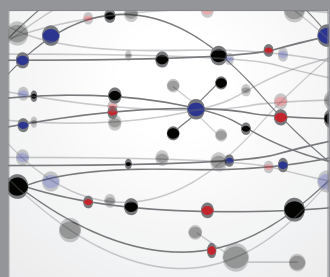

The Scientific World Journal
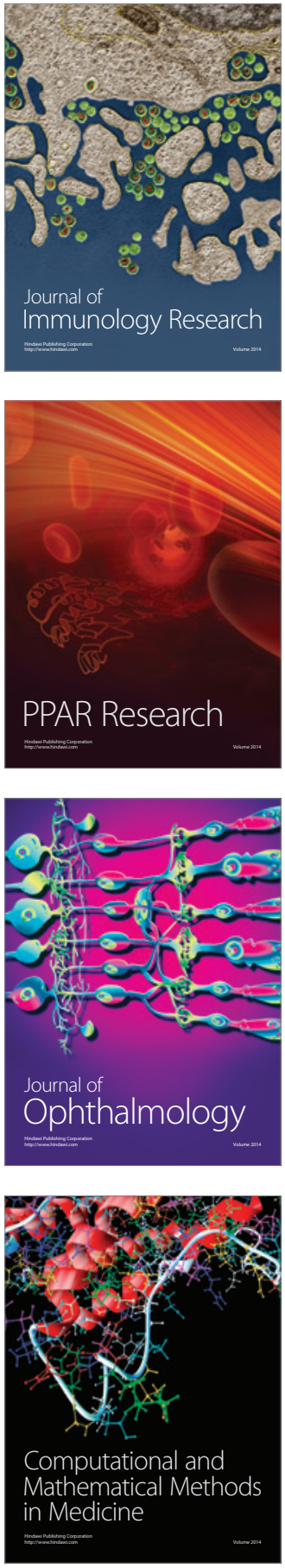

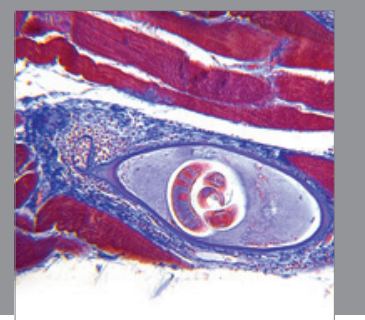

Gastroenterology

Research and Practice
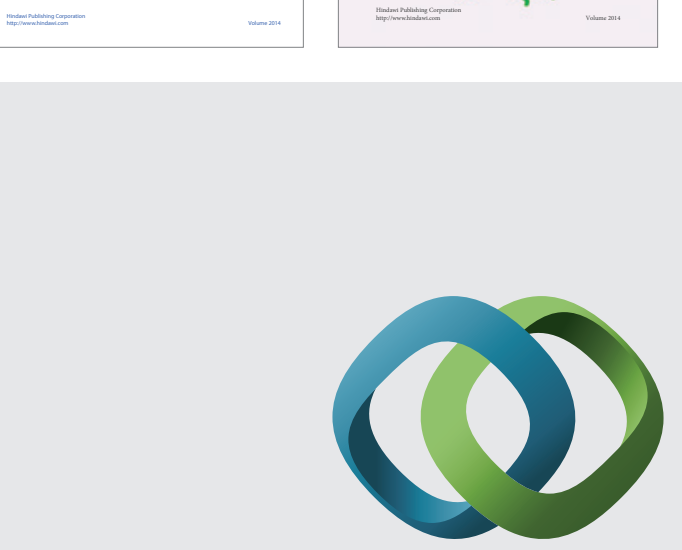

\section{Hindawi}

Submit your manuscripts at

http://www.hindawi.com
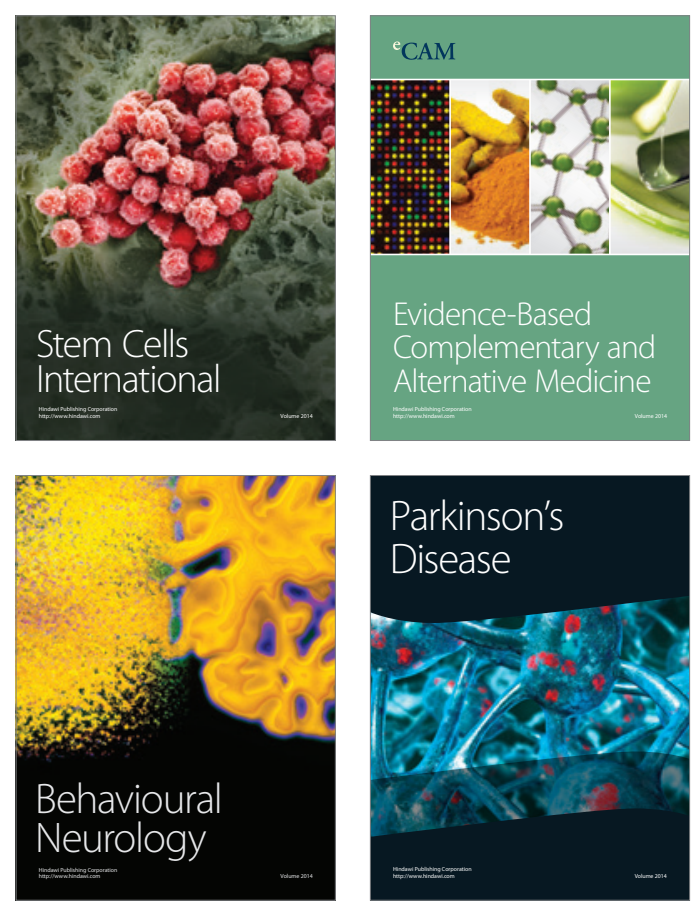

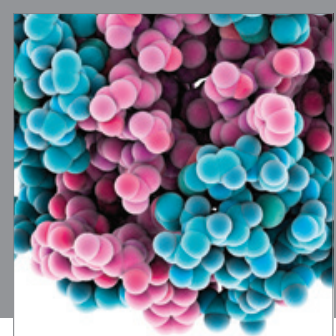

Journal of
Diabetes Research

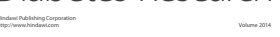

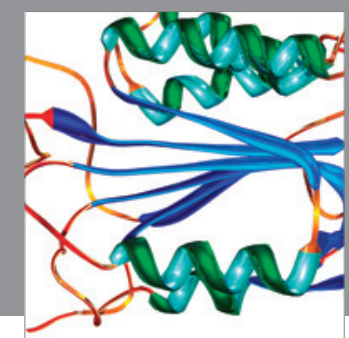

Disease Markers
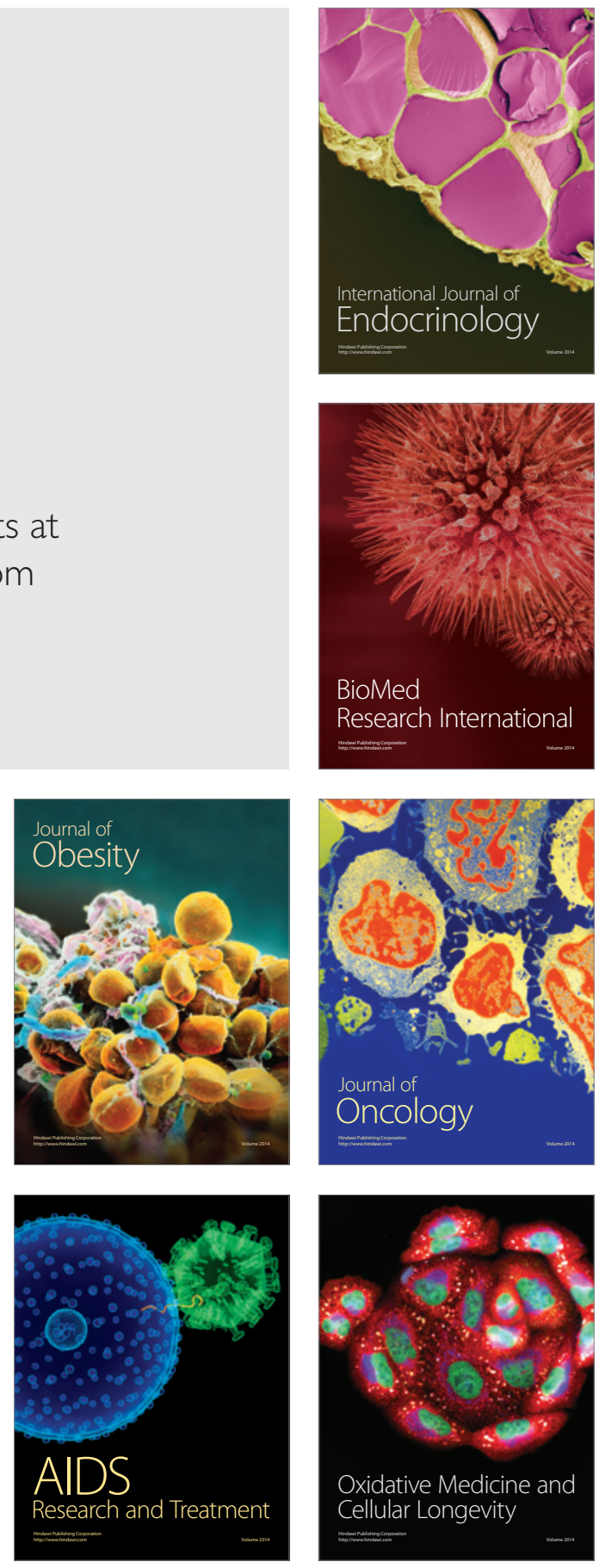